\title{
Controlling diffusion for a self-healing radiation tolerant nanostructured ferritic alloy
}

\author{
M. K. Miller ${ }^{1}$, C. M. Parish ${ }^{2}$ and H. Bei ${ }^{2}$ \\ ${ }^{1}$ Center for Nanophase Materials Sciences \\ Oak Ridge National Laboratory, \\ Oak Ridge, TN 37831, USA \\ ${ }^{2}$ Materials Science and Technology Division, \\ Oak Ridge National Laboratory, \\ Oak Ridge, TN 37831, USA
}

Contact information:

Dr. Michael K. Miller, Center for Nanophase Materials Sciences, Oak Ridge National Laboratory, P.O. Box 2008, MS 6139, Oak Ridge, TN 37831-6136, USA

E-mail: millermk@ornl.gov; Phone: (865) 574 4719; fax: (865) 2413650

This submission was sponsored by a contractor of the United States Government under contract DE-AC0500OR22725 with the United States Department of Energy. The United States Government retains, and the publisher, by accepting this submission for publication, acknowledges that the United States Government retains, a nonexclusive, paid-up, irrevocable, worldwide license to publish or reproduce the published form of this submission, or allow others to do so, for United States Government purposes. 


\begin{abstract}
Diffusion plays a major role in the stability of microstructures to extreme conditions of high temperature and high doses of irradiation. In nanostructured ferritic alloys, first principle calculations indicate that the binding energy of vacancies is reduced by the presence of oxygen, titanium and yttrium atoms. Therefore, the number of free vacancies available for diffusion can be greatly reduced. The mechanical properties of these alloys, compared to traditional wrought alloys of similar composition and grain structure, is distinctly different, and the ultrafine grained alloy is distinguished by a high number density of Ti-Y-Oenriched nanoclusters and solute clusters, which drives the mechanical response. When a displacement cascade interacts with a nanocluster, the solute atoms are locally dispersed into the matrix by ballistic collisions, but immediately a new nanocluster reforms due to the local supersaturation of solutes and vacancies until the excess vacancies are consumed. The result of these processes is a structural material for advanced energy systems with a microstructure that is self-healing and tolerant to high doses of radiation and high temperatures.
\end{abstract}

\title{
Keywords
}

Diffusion, trapping, vacancies, radiation damage; atom probe tomography, ion irradiation 


\section{Introduction}

As energy is humanities' most pressing problem in the foreseeable future, new materials with greatly improved properties for the production of energy are becoming a critical concern. The next generation of advanced energy systems will require materials for fusion and fission reactors that can operate under extreme environments of high temperature and high dose. Typical parameters are an operating temperature of up to $\sim 700{ }^{\circ} \mathrm{C}$ and a maximum dose at end of life of up to $\approx 500$ displacements per atom (dpa) [1]. Therefore, materials for these environments require good mechanical properties at elevated temperatures, resistance to long term creep, and high tolerance to radiation damage. Structural steels have limited high temperature properties, due to a combination of grain growth and precipitate coarsening well below this temperature range, even without the additional radiation component which further accelerates diffusion by the introduction of vacancies and self-interstitial atoms as the result of displacement cascades.[2,3] In steels, these processes are controlled by the diffusion of vacancies, interstitial carbon atoms, and the alloying elements at these elevated temperatures.

Therefore, a different approach is required that better controls the diffusion processes than in the Fe-C-X systems used in all steels. One viable approach is to control the diffusion of vacancies through the introduction of a high density of strong trapping sites. Ideally, the number of available trapping sites should exceed the number of excess vacancies that are produced during the service life of the reactor. The Fe-O-X system is a potential candidate, as the binding energy of vacancies with oxygen is significantly higher than that with carbon [4]. This system is exploited in oxide dispersion strengthened (ODS) steels, and, in particular, the sub-category of nanostructured ferritic alloys (NFA) [5-16]. As the accumulation of vacancies at high doses will be suppressed by the trapping of vacancies at solute clusters, nanoclusters and precipitates, void swelling in high dose neutron-irradiated materials will also be minimized [17,18]. Similarly, creep and irradiation-driven creep will also be suppressed, if there is limited number of free vacancies present. Although it is not currently experimentally possible to detect individual vacancies or 
vacancy-solute clusters by any microscopy technique, previous positron annihilation spectroscopy has indicated that vacancies are an important component in these materials [19]. In addition, the stability of the microstructure after exposure to extreme environments can indirectly provide evidence of the effectiveness of vacancy trapping. Recently, the availability of single atom detectors with detection efficiencies of greater than $80 \%$, have enabled atom probe tomography (APT) to detect and quantify solute clusters containing between 2 and 9 atoms in NFAs [20].

Because the solubility limit of oxygen in iron is only a few parts per million, traditional casting methods cannot be used to produce Fe-O-X alloys with significant oxygen contents in the ferrite matrix. In cast FeO-X alloys, almost all of the oxygen in solution in the liquid phase precipitates out as coarse oxides upon cooling, which do not confer the required mechanical properties. Therefore, mechanically alloying is an alternative method that can be used to produce such alloys.

In order to verify this new diffusion-controlled concept through vacancy trapping, a mechanically-alloy 14YWT NFA has been characterized by a combination of APT and transmission electron microscopy in the unirradiated condition and after high-dose heavy-ion irradiation at temperatures between $-100{ }^{\circ} \mathrm{C}$ and $750{ }^{\circ} \mathrm{C}$. Most importantly, the mechanical-alloyed flakes contained a high concentration of oxygen in the ferrite matrix ( 0.4 at. \%). This high-oxygen alloy was selected as it is known to exhibit excellent high temperature creep properties $[21,22]$, and high tolerance to high doses of neutron irradiation and extreme doses of ion irradiation [23]. As this alloy is produced by ball milling a master alloy together with yttria

powder, the primary solutes $(\mathrm{Cr}, \mathrm{W}, \mathrm{Ti}$, and $\mathrm{Y})$ in the resulting flakes are also distributed into a solid solution. It is not possible to solution treat these alloys at high temperatures to produce a uniform solute distribution, or relax the stresses introduced by mechanical alloying without adversely affecting the microstructure and its excellent stability for creep resistance and radiation tolerance.

\section{Experimental}

The nominal composition of the 14YWT NFA used in this study was Fe- 13.9 at. \% Cr, $0.16 \% \mathrm{~W}, 0.24 \%$ Ti, $0.14 \% \mathrm{Y}$, and $0.39 \% \mathrm{O}$ [12]. The mechanically-alloyed flakes were canned in mild steel, consolidated 
into bulk form by a hot extrusion process at $850{ }^{\circ} \mathrm{C}$, and then annealed for $1 \mathrm{~h}$ at $1000{ }^{\circ} \mathrm{C}$. This material was characterized in the unirradiated condition, and after $\mathrm{Au}$ and $\mathrm{Pt}$ ion irradiations to doses up to $\approx 150$ dpa at temperatures between $-100{ }^{\circ} \mathrm{C}$ and $750{ }^{\circ} \mathrm{C}$.

Atom probe tomography [24] characterizations were performed in voltage-pulsed mode with a CAMECA Instruments Inc. LEAP 4000X HR local electrode atom probe [24,25]. The operating parameters used were a specimen temperature of $50 \mathrm{~K}$, a pulse repetition rate of $200 \mathrm{kHz}$, and a pulse ratio of 0.2 . Atom probe specimens for the irradiated materials were extracted by an in-situ lift-out (INLO) focussed-ionbeam-based method [26] at a specific depth from the implantation surface [20] corresponding to a desired dose. The doses were estimated from the Stopping and Range of Ions in Materials (SRIM) [27] for the implantation conditions with the method of Stoller et al. [28]. The average effective radii of the nanoclusters and precipitates (excluding the low volume fraction $\operatorname{Ti}(\mathrm{O}, \mathrm{C}, \mathrm{N})$ ) were estimated from the averages of the volumes defined by the Ti concentration isosurface, i.e., $r_{e f f}=\sqrt[3]{3 V / 4 \pi}$, assuming a spherical geometry. The number densities were estimated from the number of fully-contained precipitates plus half the partially-contained nanoclusters in the volume, the number of ions in the assigned mass peaks, ignoring $\mathrm{Ga}$ and the hydrogen peaks, taking into account the detection efficiency of the mass spectrometer, 37\%, and the volume of the unit cell of Fe [24]. The error bar given is the standard deviation, $s=\sqrt{\sum\left(x_{i}-\bar{x}\right)^{2} / n-1}$, of the measurements, where $x_{i}$ are the estimated effective radii, $r_{\text {eff }}, \bar{x}$ the average of the measurements, and $n$ the number of observations.

Combined energy dispersive spectroscopy (EDS) and electron energy loss spectroscopy (EELS) spectrum images were acquired with a Philips CM200FEG SuperTwin scanning/transmission electron microscope (STEM). Detectors were an EDAX conventional Si(Li) X-ray detector and a Gatan Imaging Filter model 678, operated in EELS mode. STEM spectrum imaging was performed at $200 \mathrm{keV}$ with $1.5-2.0 \mathrm{~nm}$ diameter, 1-2 nA probes. Beam control and data acquisition was performed with the use of a Gatan Digiscan controller and Digital Micrograph software, with active drift compensation. The TEM 
specimens were also fabricated by an INLO method. The grain boundaries were image in a near edge-on orientation.

A Nanoindenter-XP system with Berkovich tip was used to measure the hardness and modulus of both ion irradiated and unirradiated materials at room temperature. Load (P) and displacement (h) were continuously recorded up to a maximum applied load $\left(\mathrm{P}_{\max }\right)$ of $1.5 \mathrm{mN}$ at a constant indentation strain rate $(\mathrm{dP} / \mathrm{dt}) / \mathrm{P}$ of $0.05 \mathrm{~s}^{-1}$, which results in a maximum depth of $\sim 100 \mathrm{~nm}$. The indentation depths are approximately one tenth of the thickness of the irradiated region, thus minimizing the influence of the unirradiated material underneath the irradiated layer. As this extended region spanned different regions of the dose profile, a precise dose in dpa cannot be accurately quoted. Therefore, fluences and the maximum dose in dpa are quoted instead for the hardness results. The hardness and modulus were determined from the P-h unloading curves by the Oliver-Pharr method [29].

\section{Results and discussion}

Several studies of the microstructure of the unirradiated condition of this 14YWT NFA have been reported previously $[9,11-18]$, so only the microstructural features pertinent to this study are reported here. In the unirradiated condition, the simultaneous EDS and EELS results, Fig. 1, indicate that the levels of $\mathrm{Cr}$ and $\mathrm{W}$ appear to be uniformly enriched along the grain boundary. APT results indicate solute segregation of $\mathrm{Cr}$ and $\mathrm{W}$ to the grain boundaries with local variations depending on the local curvature and the location of precipitates on the boundary [14]. The simultaneous EDS and EELS results also revealed that the Ti and Y levels appear to have some local enrichment on the grain boundaries. The reason for the Ti and Y non-uniformity is apparent in the APT results by the presence of fine Ti-, Y-and O-enriched precipitates along the grain boundaries [14], as shown below. APT also revealed that a high number density, $\sim 6.9( \pm 0.6) \times 10^{23} \mathrm{~m}^{-3}$, of Ti-, Y- and O-enriched spheroidal nanoclusters are formed in the interiors of the grains [11], as shown in Fig. 2. Their size distribution is shown in Fig.3 and the associated parameters of this distribution, i.e., mean, mode, median, size range, skewness, and excess kurtosis, are listed in Table 1. 
When the extruded alloy is exposed to high doses of irradiation at low temperatures $\left(-100\right.$ to $\left.\sim 75{ }^{\circ} \mathrm{C}\right)$, the microstructure changes significantly. Both TEM results, Fig. 4, and APT atom maps, Fig. 5, for the NFA that was Pt ion irradiated to a dose of $\approx 100 \mathrm{dpa}$ at $-100{ }^{\circ} \mathrm{C}$ indicate that all the solutes in the pre-existing nanoclusters have been locally redistributed into the matrix through ballistic collisions during the production of the displacement cascades. The grain boundaries in the TEM data are indicated by the arrows in the high-angle annular dark field (HAADF) image. Similarly, there is no evidence of any precipitates, or solute segregation at the grain boundaries in Fig. 5. The dissolution of the nanoclusters increases the hardness from $8.2 \pm 0.6 \mathrm{GPa}$ in the unirradiated material to $10.3 \pm 0.8 \mathrm{GPa}$ in the irradiated layer (fluence of $6 \times 10^{16} \mathrm{Au} \mathrm{cm}^{-2}$ with a maximum dose of $\approx 225 \mathrm{dpa}$ ), and decreases the modulus from $269 \pm 28 \mathrm{GPa}$ in the unirradiated material to $251 \pm 23 \mathrm{GPa}$ at the irradiation temperature of $75^{\circ} \mathrm{C}$. These results indicate that despite the additional vacancies produced in the cascade affected zone (CAZ) by the displacement cascade, there is insufficient diffusion during the irradiation time in this low temperature range for the solutes to reform nanoclusters or grain boundary precipitates, or segregate to the grain boundaries. The time available for each re-solutionized nanocluster to reform will depend on when the displacement cascade occurred during the irradiation and the post irradiation cool down time, and could vary from minutes up to $\sim 4 \mathrm{~h}$. However, there may be sufficient diffusion after the redistribution of solute for the formation of vacancy-solute clusters of oxygen, titanium and/or yttrium. The presence of these vacancy-solute clusters and the high oxygen content could also contribute to the higher hardness compared to the unirradiated condition.

In contrast, when similar high dose irradiations are performed at significantly higher temperatures, such as the maximum intended operating temperature of these NFA, e.g., $750{ }^{\circ} \mathrm{C}$ for the NFA Pt ion irradiated to a dose of $\approx 125 \mathrm{dpa}$, the intragranular nanoclusters, Figs. 6 , were present but with a slightly reduced, but not statistically significant, number density of $\sim 5.8( \pm 1.0) \times 10^{23} \mathrm{~m}^{-3}$. In order to compare with the unirradiated condition, the size distribution of this irradiation condition is included in Fig. 3 and the associated parameters are listed in Table 1 . These distributions exhibit the same mean $(1.78 \mathrm{~nm})$ and 
skewness $(0.86$ and $0.88 \mathrm{~nm}$, respectively for the unirradiated and irradiated conditions). The range of sizes is larger in the unirradiated that the high temperature irradiation. However, this parameter is strongly controlled by the relatively limited data/sampling to be able to state anything conclusively (even though there are more particles in the distribution of the unirradiated). The mode and excess kurtosis are different. The mode is smaller in the unirradiated condition, possibly when taken together with the reduction in number density indicating that the precipitates regrow to a larger size during irradiation at $750{ }^{\circ} \mathrm{C}$. In addition, the excess kurtosis indicate that size distribution of the particles in irradiated condition are close to that of a normal/Gaussian distribution, i.e., 0 , whereas, the size distribution in the unirradiated condition is moderately more peaked than a normal distribution. In addition, the grain boundary segregation of $\mathrm{Cr}$ and $\mathrm{W}$, and precipitates, Figs. 7 and 8, are present. Details of the solute segregation and precipitation at the grain boundaries has been discussed in detail previously [14,30]. These results indicate that after the solutes in individual pre-existing nanoclusters have been locally redistributed into the matrix through ballistic collisions during the production of the displacement cascades, there is now sufficient diffusion to re-precipitate the nanoclusters through the additional vacancies produced during the cascade and the higher temperature.

This diffusion-controlled process at elevated temperatures, as illustrated in Fig. 9, would take place on a local scale approximately equal to the size of the CAZ, which was estimated from SRIM to be $\sim 5.4 \mathrm{~nm}$ in diameter. The solute in each nanocluster is redistributed during high-temperature irradiation, and then a new nanocluster would nucleate close to its original position, due to the localized enhanced diffusion. Coarsening would continue until all the free vacancies are consumed either in the new nanocluster, its neighbors, at vacancy-solute clusters of oxygen, titanium and/or yttrium in the ferrite matrix, or in recombination with the newly-generated self-interstitial atoms. Only a small number of nanoclusters would be in this transient state at any given instant of time. The difference between this mechanism for the irradiated condition and the normal nucleation and growth of the nanoclusters (until the vacancies are consumed) during the soak and extrusion process may explain the difference in the kurtosis in the size 
distribution. More importantly, this alloy would be self-healing during service in the operational temperature range of future advanced reactors. In addition, the mechanical properties of the NFA would not be significantly compromised, as the distribution of the nanoclusters only coarsens slightly in agreement with long-term thermal aging results.

\section{Conclusions}

This combined APT and TEM study has indicated that the solutes in nanoclusters present and Cr and $\mathrm{W}$ segregated at the grain boundary in the unirradiated 14YWT nanostructured ferritic alloy are redistributed in the solid solution during high dose ion irradiation at $-100{ }^{\circ} \mathrm{C}$. In contrast, when the ion irradiation was performed close to the intended maximum service operation temperature at $750{ }^{\circ} \mathrm{C}$, the nanoclusters were present, and segregation of $\mathrm{Cr}$ and $\mathrm{W}$ at the grain boundaries was evident. It was proposed that a diffusion-controlled process takes place on a local scale, in which the solute atoms in each nanocluster is initially redistributed into the solid solution during high-temperature ion irradiation, and one or more new nanoclusters would nucleate close to its original position, due to the localize enhanced diffusion, and growth would continue until all the free vacancies are consumed. The high stability and radiation tolerance of this Fe-O-X alloy is due to the strong binding between the vacancies and the oxygen, titanium and yttrium atoms.

\section{Acknowledgements}

This research was sponsored by the Materials Sciences and Engineering Division, Office of Basic Energy Sciences, US Department of Energy. The microscopy research was conducted at the Center for Nanophase Materials Sciences, which is a DOE Office of Science User Facility. Ion irradiations were performed at the Environmental Molecular Sciences Laboratory (EMSL), a national scientific user facility sponsored by the US Department of Energy's Office of Biological and Environmental Research and located at Pacific Northwest National Laboratory. The 14YWT material used in this study was provided by Dr. D. T. Hoelzer, ORNL. The heavy ion irradiations were performed by Dr. Y. Zhang, ORNL. 


\section{References}

[1] S.J. Zinkle, J.T. Busby, Mater. Today, 12(11) (2009) 12-19; S.J. Zinkle, and G. S. Was, Acta Mater. 61(3) (2013) 735-758.

[2] R.S. Averback and D.N. Seidman, "Energetic Displacement Cascades and Their Roles in Radiation Effects," Materials Science Forum 15-18 (1987) 963-984.

[3] D.N. Seidman, R.S. Averback, and R. Benedek, "Displacement Cascades: Dynamics and Atomic Structure," Physica Status Solidi (b) 144 (1987) 85-104.

[4] C.L. Fu, Private communication

[5] R.L. Klueh, J. Nucl. Mater., 378(2) (2008) 159-166.

[6] K.L. Murty, I. Charit, J. Nucl. Mater., 383 (2008) 189-195.

[7] G.R. Odette, M.J. Alinger, B.D. Wirth, Annual Review of Materials Research, 38 (2008) 471-503.

[9] M.K. Miller, E.A. Kenik, K.F. Russell, L. Heatherly, D.T. Hoelzer, P.J. Maziasz, Mater. Sci. Eng. A., A353 (2003) 140-145.

[10] M.J. Alinger, G.R. Odette, D.T. Hoelzer, Acta Mater., 57(2) (2009) 392-406.

[11] M.K. Miller, D.T. Hoelzer, K.F. Russell, Mater. Sci. Forum, 654-656 (2010) 23-28.

[12] M.K. Miller, C.M. Parish, Mater. Sci. Technol., 27 (2011) 729-734

[13] M.K. Miller, D.T. Hoelzer, J. Nucl. Mater., 418 (2011) 307-310.

[14] M.K. Miller, L. Yao, Current Opinions in Solid State and Materials Science, 17 (2013) 203-210.

[15] A. Certain, S. Kuchibhatla, V. Shutthanandan, D.T. Hoelzer, T.R. Allen, J. Nucl. Mater., 434 (2013) 311-321.

[16] J. He, F. Wan, K. Sridharan, T.R. Allen, A. Certain, V. Shutthanandan, Y.Q. Wu, J. Nucl. Mater., 455 (2014) 41-45.

[17] C.L. Fu, M. Krcmar, G.S. Painter, X.-Q.Chen , Phys. Rev. Lett., 97 (2007) 225502.

[18] M.K. Miller, C.L. Fu, M. Krcmar, D.T. Hoelzer, C.T. Liu, Front. Mater. Sci. China, 3(1) (2009) 914. 
[19] J. Xu, C.T. Liu, M.K. Miller, H. Chen, Phys. Rev. B, 79 (2009) 020204-1-4 DOI:

10.1103/PhysRevB.79.020204.

[20] M.K. Miller, D. Reinhard and D.J. Larson, J. Nucl. Mater., submitted to this issue.

[21] R.L. Klueh, J. Nucl. Mater., 378(2) (2008) 159-166.

[22] J.H. Schneibel, C.T. Liu, M.K. Miller, M.J. Mills, P. Sarosi, M. Heilmaier and D. Sturm, Scr. Mater., 61(8) (2009) 793-796.

[23] M K. Miller, Y. Zhang, Ultramicroscopy, 111 (2011) 672-675.

[24] M.K. Miller, R. G. Forbes, Atom Probe Tomography: The Local Electrode Atom Probe, Springer, New York NY, 2014.

[25] D.J. Larson, T.J. Prosna, R.M. Ulfig, B.P. Geiser, T. F. Kelly, Local Electrode Atom Probe Tomography, Springer, New York NY, 2013.

[26] M.K. Miller, K.F. Russell, K. Thompson, R. Alvis and D.J. Larson, Microscopy and Microanalysis, 13(6) (2007) 428-436.

[27] J.F. Ziegler, J.P. Biersack, M.D. Ziegler, SRIM: The Stopping and Range of Ions in Matter, Lulu Press, Morrisville, 2008.

[28] R.E. Stoller, M.B. Toloczko, G.S. Was, A.G. Certain, S. Dwaraknath, F.A. Garner, Nucl. Instrum. Methods Phys. Res. Section B-Beam Interact. Mater. Atoms, 310 (2013) 75-80.

[29] W.C. Oliver, G.M. Pharr, J. Mater. Res., 19 (2004) 3-20.

[30] E. A. Marquis, S. Lozano-Perez, V. de Castro, Journal of Nuclear Materials, 417 (1-3) (2011) $257-$ 261. 
Table 1. Parameters of the particle size distributions for the14YWT NFA in the unirradiated condition and after irradiation at $750{ }^{\circ} \mathrm{C}$. All estimates are given in $\mathrm{nm}$.

\begin{tabular}{|l|l|l|}
\hline Parameters & Unirradiated & Irradiated at $750^{\circ} \mathrm{C}$ \\
\hline Mean & $1.78 \pm 0.74$ & $1.78 \pm 0.90$ \\
\hline Mode (most numerous) & 1.24 & 1.74 \\
\hline Median (middle number) & 1.70 & 1.57 \\
\hline Minimum & 0.39 & 0.57 \\
\hline Maximum & 5.71 & 4.55 \\
\hline Skewness (degree of asymmetry) & 0.88 & 0.86 \\
\hline Excess Kurtosis (peakedness) & 1.38 & 0.06 \\
\hline
\end{tabular}




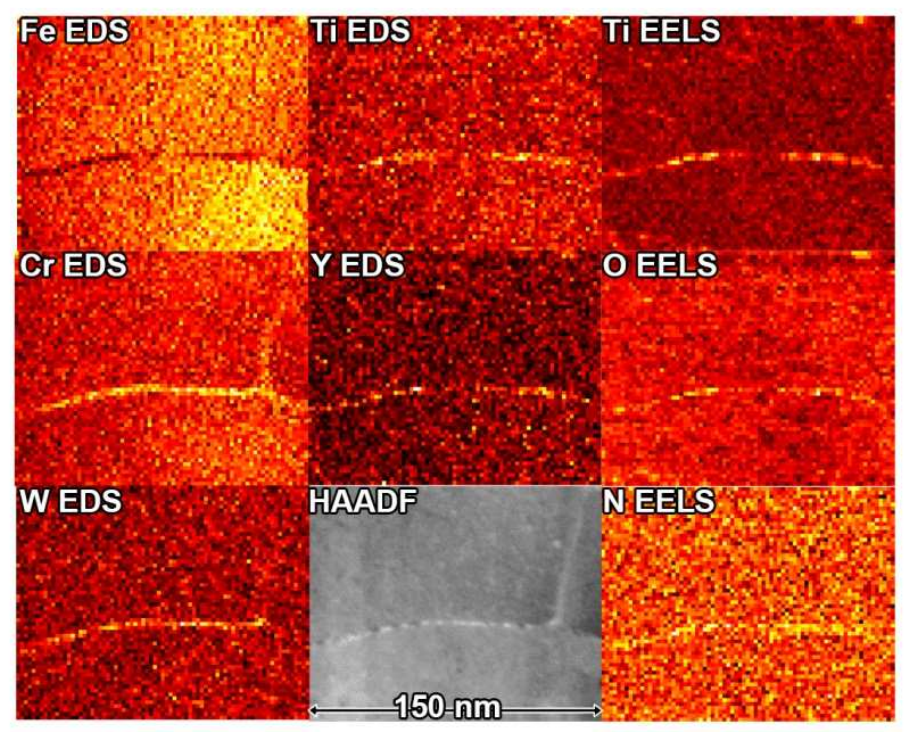

Fig. 1. The solute distribution found in the microstructure of the 14YWT NFA in the as-extruded and aged $1 \mathrm{~h}$ at $1000{ }^{\circ} \mathrm{C}$ base unirradiated condition. The HAADF image indicates the positions of 2 grain boundary segments viewed in a near edge-on orientation, and the EDS and EELS maps revealed enrichments of $\mathrm{Cr}, \mathrm{W}, \mathrm{Ti}, \mathrm{Y}, \mathrm{O}$ and $\mathrm{N}$ due to a combination of solute segregation and precipitation. 


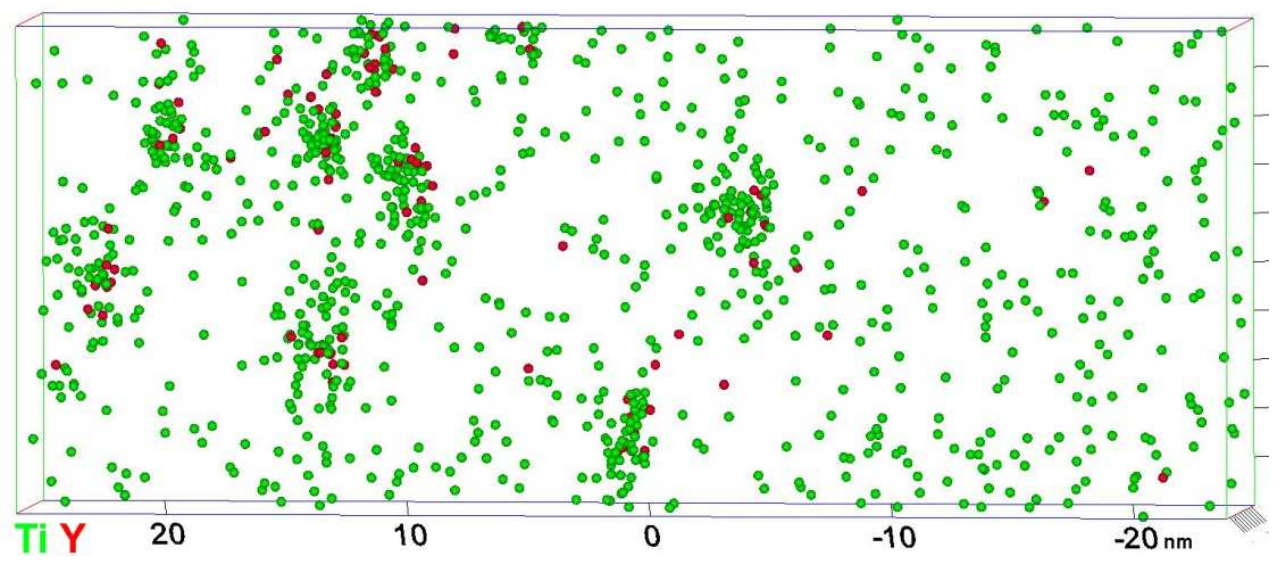

Fig. 2. Atom map of the solute distribution found in the microstructure of the 14YWT NFA in the asextruded and aged $1 \mathrm{~h}$ at $1000{ }^{\circ} \mathrm{C}$ base unirradiated condition. A high number density of 2-nmdiameter nanoclusters are evident in the ferrite matrix. 


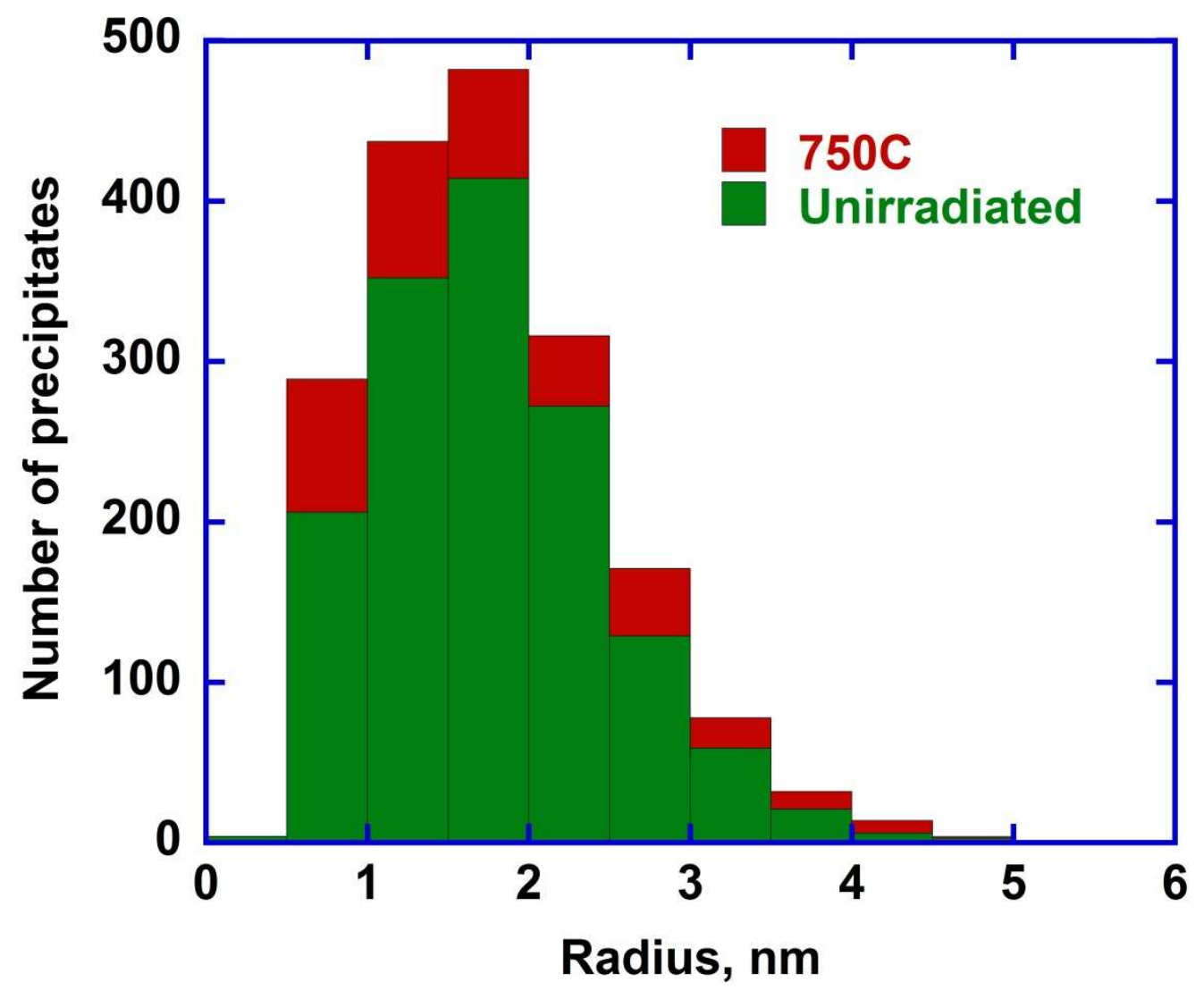

Fig. 3.The particle size distributions for the14YWT NFA in the unirradiated condition and after irradiation at $750{ }^{\circ} \mathrm{C}$. 


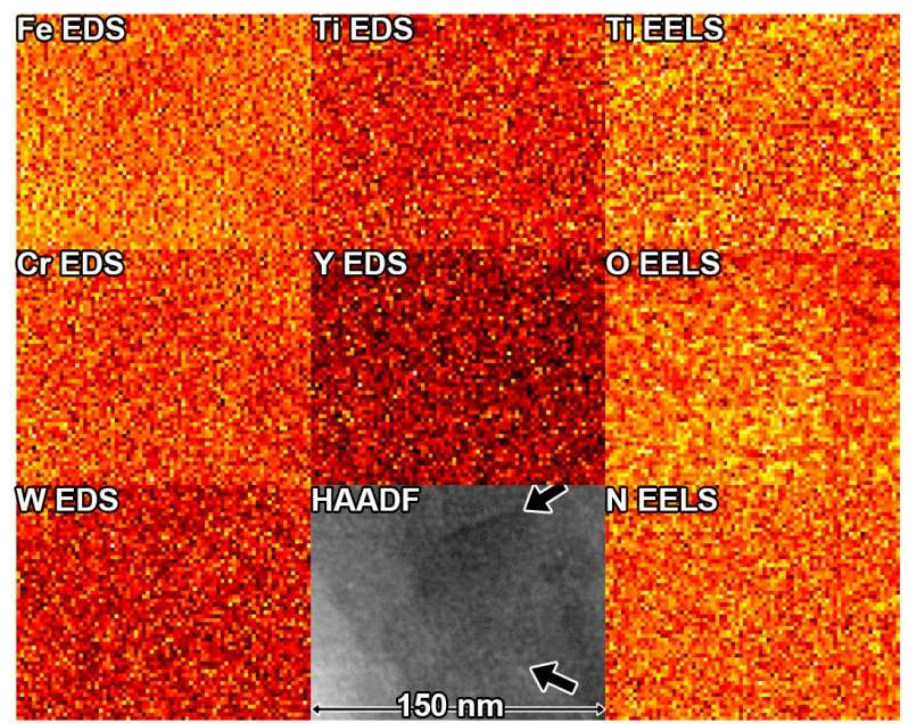

Fig. 4. A uniform distribution of solute was found in the microstructure in the HAADF image and by EDS and EELS after Pt ion irradiation to a dose of $\approx 100$ dpa at $-100{ }^{\circ} \mathrm{C}$. Arrows in the HAADF image indicate the position of grain boundaries. 


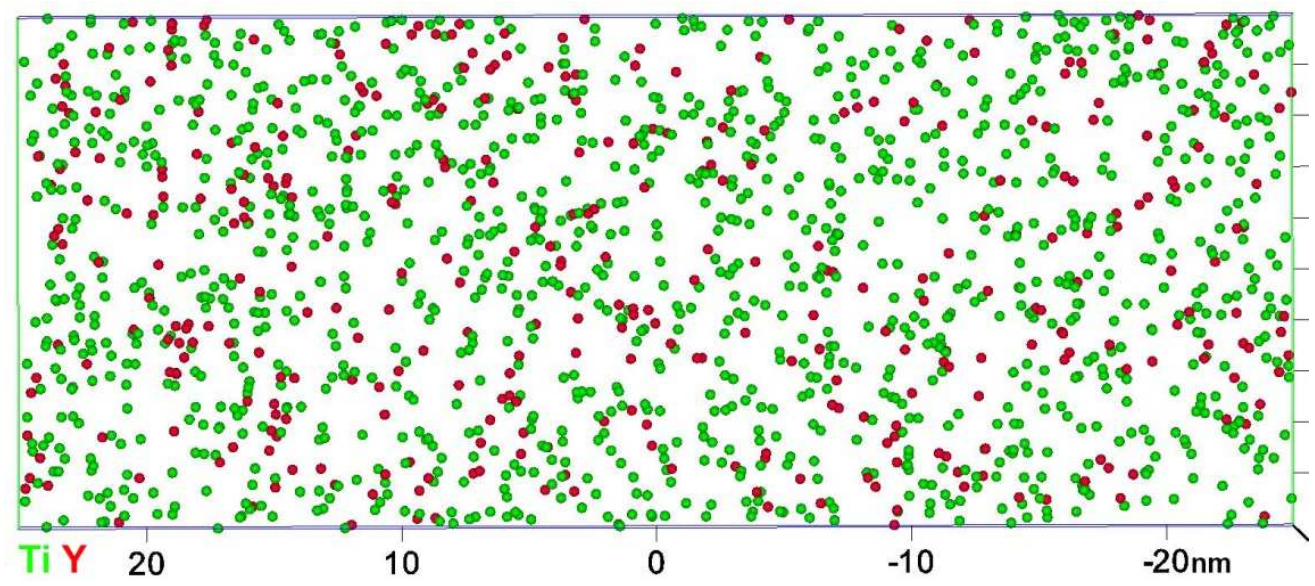

Fig.5. This 10-nm-thick section of an atom map revealed that the solute atoms in the NCs have been ejected into a solid solution after Pt ion irradiation to a dose of $\approx 100$ dpa at $-100{ }^{\circ} \mathrm{C}$. 


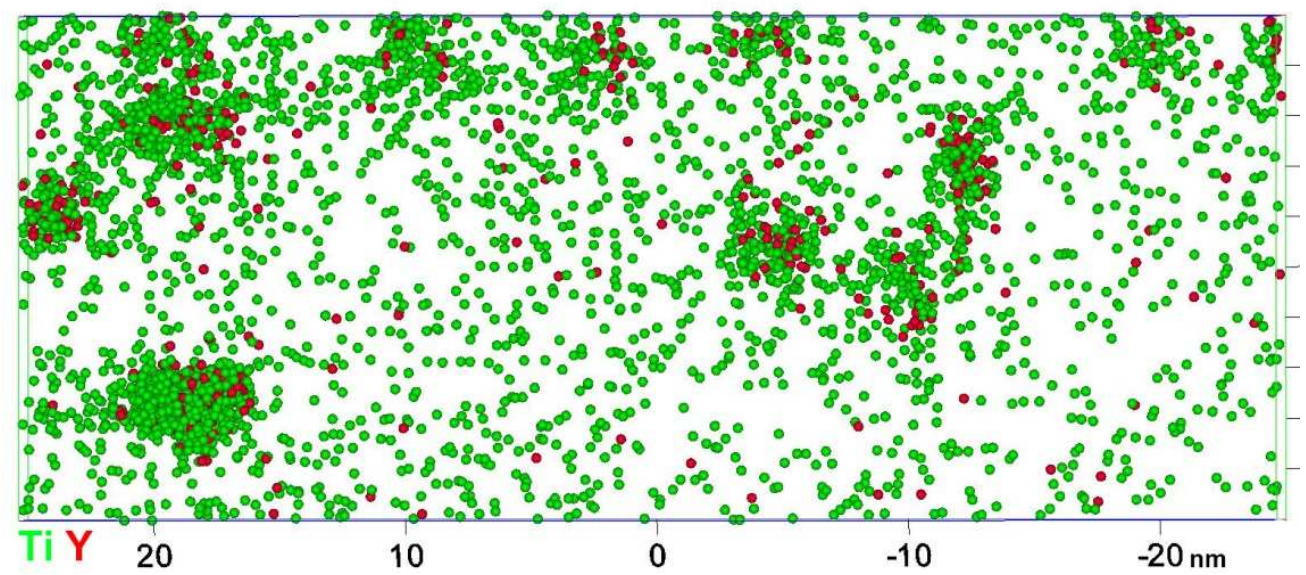

Fig. 6. 10-nm-thick section atom map of the Ti- and Y-enriched 2.5-nm-diameter nanoclusters in the ferrite matrix after Pt irradiation to a dose of $\approx 150$ dpa at $750{ }^{\circ} \mathrm{C}$. 


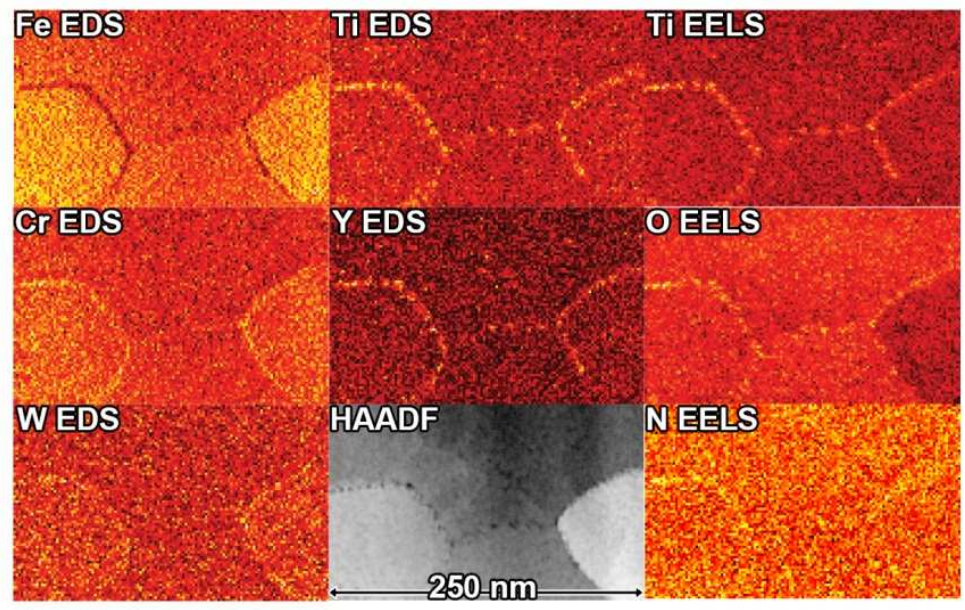

Fig. 7. After Pt irradiation to a dose of $\approx 150 \mathrm{dpa}$ at $750{ }^{\circ} \mathrm{C}$, the HAADF image indicates the presence of precipitates on the grain boundary, and the EDS and EELS maps indicated that the grain boundaries are decorated with $\mathrm{Cr}, \mathrm{W}, \mathrm{Ri}, \mathrm{Y}, \mathrm{O}$ and $\mathrm{N}$ atoms. 


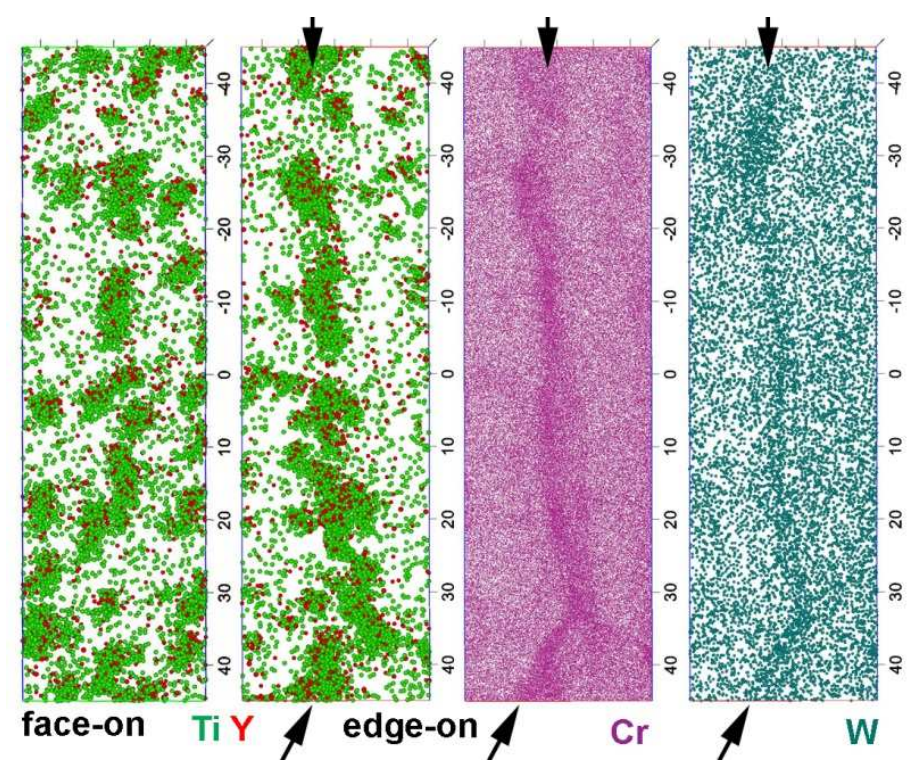

Fig. 8. 10-nm-thick section atom map of the Ti- and Y-enriched nanoclusters (viewed face-on and edgeon) and $\mathrm{Cr}$ and $\mathrm{W}$ segregation to a grain boundary after Pt irradiation to a dose of $\approx 150 \mathrm{dpa}$ at 650 ${ }^{\circ} \mathrm{C}$. 


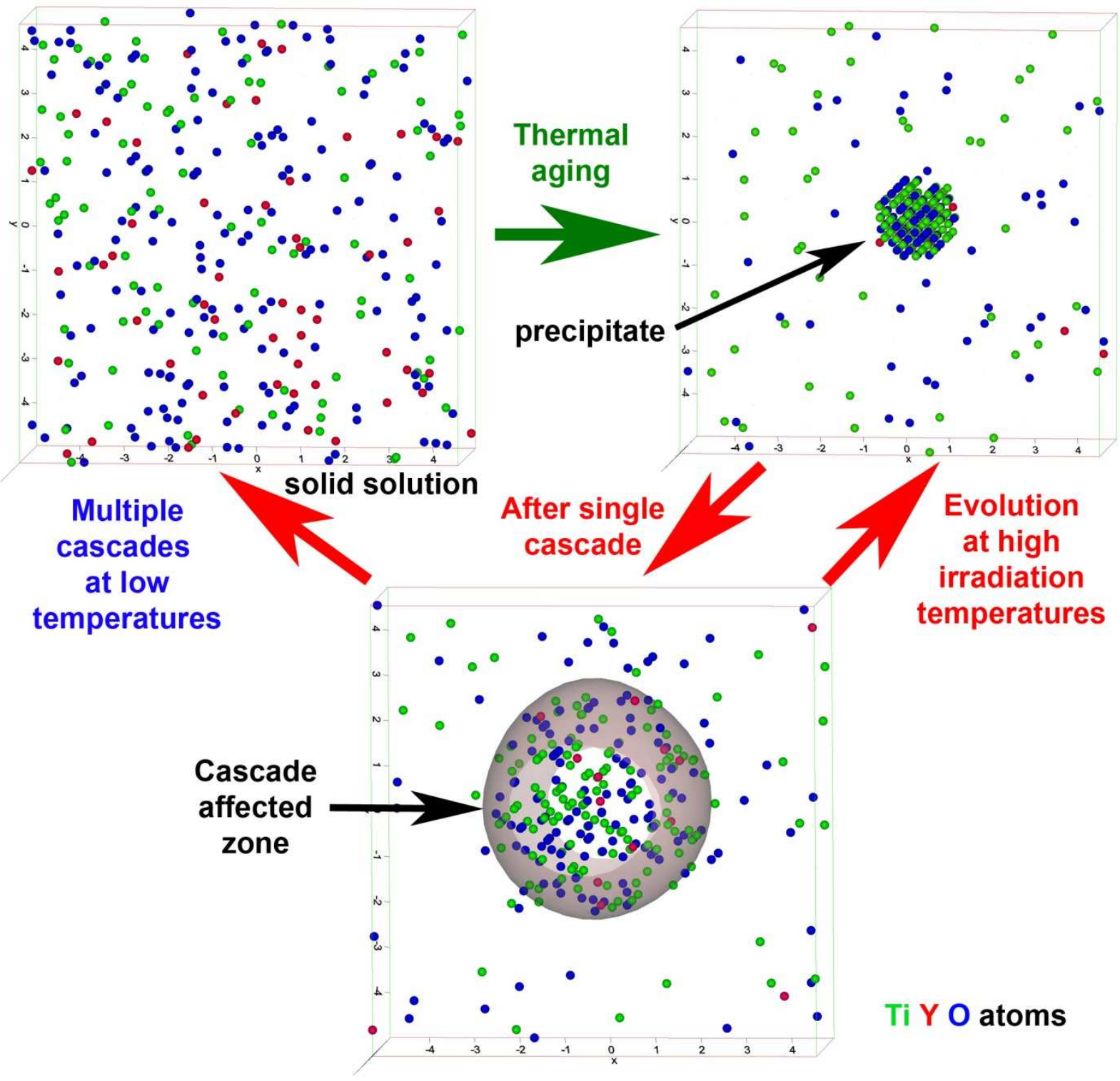

Fig. 9. Schematic diagram of the dissolution and re-precipitation process than occurs during high dose irradiation, e.g., 100-150 dpa, at temperatures above $\sim 350{ }^{\circ} \mathrm{C}$. 
Table 1. Parameters of the particle size distributions for the14YWT NFA in the unirradiated condition and after irradiation at $750{ }^{\circ} \mathrm{C}$. All estimates are given in $\mathrm{nm}$.

\begin{tabular}{|l|l|l|}
\hline Parameters & Unirradiated & Irradiated at $750^{\circ} \mathrm{C}$ \\
\hline Mean & $1.78 \pm 0.74$ & $1.78 \pm 0.90$ \\
\hline Mode (most numerous) & 1.24 & 1.74 \\
\hline Median (middle number) & 1.70 & 1.57 \\
\hline Minimum & 0.39 & 0.57 \\
\hline Maximum & 5.71 & 4.55 \\
\hline Skewness (degree of asymmetry) & 0.88 & 0.86 \\
\hline Excess Kurtosis (peakedness) & 1.38 & 0.06 \\
\hline
\end{tabular}

